\title{
Psychiatric comorbidity in chronic urticaria patients: a systematic review and meta-analysis
}

\author{
Gerasimos N. Konstantinou ${ }^{1+}$ and George N. Konstantinou ${ }^{2^{*+}}$ (D)
}

\begin{abstract}
Background: Dermatological illness can affect the quality of life and may coexist with psychiatric disorders.

Objective: The aim of this review was to systematically evaluate the published evidence of any psychiatric disorders that may coexist with chronic urticaria (CU) and any effect psychiatric interventions may have on CU.

Methods: Following the Cochrane guidance, we conducted a systematic literature search using web-based search engines provided by PubMed (for Medline database), Google Scholar and Scopus for studies that have investigated the existence of psychiatric comorbidity in patients with CU. To be included, a study had to possess features, such as: (1) distinction between chronic urticaria and allergic conditions, (2) direct collection of diagnostic psychiatric data by using clinical interview and standardized questionnaires, (3) International Classification of Disorders criteria or the Diagnostic and Statistical Manual of Mental Disorders criteria for the diagnosis of mental disorders, and (4) manuscripts written or published in the English language. Unpublished research and research in progress were not included. All the eligible studies were scrutinized for any reported psychiatric interventions that had any effect on CU. The systematic review has been registered on PROSPERO (registration number CRD42019122811) and was conducted following the Preferred Reporting Items for Systematic Reviews and Meta-Analysis (PRISMA).

Results: Twenty-five studies were identified. Almost one out of three CU patients have at least one underlying psychiatric disorder. None of the studies clarified whether the psychiatric disorders pre-existed the CU onset, and no association was found between CU severity and duration, and psychological functioning. Only one case report and two case series mentioned that treatment of psychiatric disorders with either anti-depressants, anti-anxiety drugs or psychological interventions might result in improvement of urticaria.

Conclusions: Patients with CU frequently experience psychiatric disorders. This highlights the need for a multidisciplinary therapeutic approach involving prompt recognition and management of any potential psychiatric disorder in addition to urticaria treatment. Further studies are needed to assess whether psychiatric disorders coexist with CU independently or follow urticaria onset and whether any psychological or psychiatric intervention may help in CU control.
\end{abstract}

Keywords: Psychiatric disorders, Psychopathology, Prevalence, Anxiety, Depression, Stress

\footnotetext{
*Correspondence: gnkonstantinou@gmail.com

${ }^{\dagger}$ Gerasimos N. Konstantinou and George N. Konstantinou contributed equally to this work

2 Department of Allergy and Clinical Immunology, 424 General Military

Training Hospital, 11 Eleftheriou Benizelou Street, Kalamaria, 55

133 Thessaloniki, Greece

Full list of author information is available at the end of the article
} 


\section{Introduction}

Chronic urticaria (CU) is a troublesome entity that presents with wheals, angioedema or both almost daily for at least 6 consecutive weeks. Both wheals and angioedema are characterized by superficial dermal swelling favoring the papillary dermis in the case of the wheal formation, and the deep dermis and subcutis in the case of the angioedema. The urticarial lesions may be intensively pruritic, and the angioedema may present with mild pain and burning sensation [1], symptoms that affect the patient's quality of life significantly and may account for stress, sleep disorders, negative self-image, disability in social functions and adverse emotions such as anger and low mood/sadness [2-4]. The prevalence of CU in the general population has been estimated to range from 0.5 to $5 \%$ and has consequently been considered as a nonnegligible clinical entity [5].

The psychological effects that several dermatological entities and allergic conditions may cause have long been speculated or even recognized [6-9]. Back in 1940, Clarke [10] lectured on the interhospital conference held at the Utica State Hospital at Utica, NY (sic) "The mental effects of allergy have received little attention, although nervous symptoms are so common in association with the allergic diseases that until recently asthma, urticaria, angioneurotic edema, and migraine were thought to be primarily diseases of the nervous system". More recently published evidence suggests a clear association between $\mathrm{CU}$ and psychiatric disorders, most commonly depression and anxiety [11-13].

Most of the physicians that consult CU patients are not aware that urticaria and several psychological or psychiatric disorders may be interconnected, except urticaria experts that approach these patients in the context of a bio-psycho-social model $[14,15]$. In the absence of any existing systematic review of the literature approaching this fact, we performed a systematic review of the literature with the intention to examine the prevalence of any psychiatric disorder among patients with $\mathrm{CU}$, any potential association that may exist and the role any psychiatric intervention may have in urticaria control and treatment. Furthermore, we investigated the literature for evidence suggestive of whether psychiatric disorders pre-exist, coexist or follow $\mathrm{CU}$ and if there is any association between $\mathrm{CU}$ severity and duration or severity of the psychological functioning.

\section{Method}

This systematic literature review was conducted and reported following the Preferred Reporting Items for Systematic Reviews and Meta-Analysis (PRISMA) [16]. The Cochrane guidance for non-randomized studies was followed for meta-analysis of the results. The protocol of this systemic review has been registered on PROSPERO with registration number CRD42019122811.

An a priori set of inclusion criteria was predefined. To be included, a study had to possess features, such as: (1) clear distinction between urticaria and other allergies, (2) clear distinction between acute and chronic urticaria, (3) utilization of appropriate clinical interviews, standardized questionnaires and criteria for psychopathology diagnosis and standard diagnostic nomenclature for mental and behavioural disorders [including the International Statistical Classification of Diseases and Related Health Problems (ICD) and the Diagnostic and Statistical Manual of Mental Disorders (DSM)], and (5) manuscripts written or published in English language. Unpublished research and research in progress were not included. The references of eligible publications were scrutinized to identify additional possible studies.

\section{Criteria for considering studies for this review Types of studies}

Included studies were randomized clinical trials (RCT), controlled clinical trials, cohort studies, case-control studies, case series and case reports relevant to $\mathrm{CU}$ patients (adults) that investigated the comorbidity of psychiatric disorders and symptoms.

\section{Types of participants}

The primary outcome of interest was the assessment of the prevalence of psychiatric comorbidities in $\mathrm{CU}$ patients by any validated measure. Secondary outcomes were measures of disease severity and psychiatric interventions on $\mathrm{CU}$ patients. The outcome terminologies presented in this report are those used in the original publications.

\section{Search strategy for identification of studies}

A comprehensive search strategy, including all the relevant synonyms for the central concepts, was developed. Web-based search engines provided by PubMed (for Medline database), Google Scholar and Scopus were utilized. Key phrases used for the research were: "psychiatric disorders and chronic urticaria", "psychiatric comorbidity and allergy", "psychiatry and urticaria", "depression and urticaria", "anxiety and urticaria", "psychiatric morbidity in dermatology", "urticaria and comorbidity", "psychiatry and dermatology", "psychiatry and allergology", "psychodermatology", and "stress and urticaria". The last publication month that has been systematically reviewed was December 2018.

\section{Data collection}

Two independent reviewers assessed all titles and abstracts, extracted all data, and assessed quality. A 
highly sensitive search strategy identified all published articles. The full text of all potentially eligible studies was assessed for eligibility against the predefined inclusion criteria. The reference list from these articles, including all relevant review articles, was analyzed for other potentially relevant studies not identified in the data-based search. All the eligible studies were scrutinized for any reported psychiatric interventions that had any effect on CU. All of the included studies were discussed and approved by the review team.

\section{Assessment of risk of bias in included studies}

The risk of bias tool described in the Cochrane Handbook for Systematic Reviews for Interventions was used to appraise the studies.

\section{Results}

Our search identified 154 potentially relevant published papers; 25 of them (11 cohort studies, 11 case-control studies, 2 case series, and 1 case report) satisfied our inclusion criteria and were included in our meta-analysis (Fig. 1). Some of these studies did use a control group (not necessarily comprised with healthy individuals) to compare psychopathologic comorbidities among CU patients, and some of them did not examine psychopathology as a whole but focused on specific psychiatric entities (e.g., studies that focused only on depression and did not examine the coexistence of any other psychiatric disorder) (Table 1).

The overall prevalence of any psychiatric comorbidity among CU patients independently of whether studies had or didn't have a control group was estimated to be $31.61 \%$, (data from Tables 2 and 3). The biggest study among them by Chu et al. [17] from Taiwan was based on an international database for health insurance. Because the sample in this study was large and the particularities of the examined individuals were expected to increase heterogeneity substantially, the pooled prevalence was calculated, as well, without this study and was found to be exactly the same (31.61\%).

The pooled prevalence of each psychiatric disorder among CU patients, categorized according to Diagnostic and Statistical Manual of Mental Disorders (DSM-5), are presented in Table 4 (data listed for disorders referred in at least two different published articles). The most prevalent were found to be sleep-wake disorders (36.7\%), followed by anxiety disorders (30.6\%), mood disorders (29.4\%), trauma and stressor-related disorders (17.3\%), somatic symptom and related disorders $(17.2 \%)$, obsessive-compulsive and related disorders $(9.3 \%)$ and substance-related and addictive disorders (4\%).

\section{Psychiatric comorbidities in patients with chronic urticaria (studies without a control group) Description of studies}

Seven studies examined psychiatric comorbidity in patients with $\mathrm{CU}$ without providing a control group [18-24].

Four of them clearly mentioned both the total number of included $\mathrm{CU}$ patients and how many of these patients had at least one psychiatric comorbidity (sum 479 and 126 , respectively, pooled prevalence $26.3 \%$, Table 2 ). From the remaining three studies, two mentioned the absolute number of patients suffering from specific psychiatric disorders, without clarifying whether any of these patients had more than one of them [23, 24]. Since psychiatric illnesses may very often co-exist, in these two studies, it was not possible to calculate the exact psychiatric comorbidity among the examined $\mathrm{CU}$ patients.

The last study by Picardi et al. [21] referred to the prevalence and correlates of suicidal ideation among patients with skin diseases. Although suicidal ideation is not categorized as a specific psychiatric disorder, according to DSM, it is closely related to the majority of mental illness.

\section{Main findings}

In 1981, Juhlin [18], after studying 330 patients with CU reported that $16 \%(n=53)$ of them had a psychiatric history of taking relevant medications. No individual psychiatric diagnoses were reported.

Pulimood et al. [19] determined the prevalence and nature of psychiatric morbidity among 1073 dermatological inpatients. Among them, 20 patients were suffering from $\mathrm{CU}, 15$ of whom were comorbid with psychiatric pathology.

In 2000 Picardi et al. [20] studied the prevalence of psychiatric disorders in 2579 dermatological outpatients. Among the 29 patients with $\mathrm{CU}$, the percentage of psychiatric cases was $34.5 \%(\mathrm{n}=10)$.

Although suicidal ideation is not yet an official psychiatric disorder according to DSM, Picardi et al. [21] sought to estimate the prevalence of suicidal ideation among 466 patients with dermatologic conditions and found that was present among 16 patients with urticaria (prevalence $18.8 \%)$.

Staubach et al. [22] in 2011 assessed the prevalence and spectrum of mental disorders and determined levels of emotional distress in patients with CU. A total sample of 100 patients with $\mathrm{CU}$ was screened for enrollment in this study and was investigated for mental disorders. Fortyeight of them were found to have one or more mental disorders. The most common mental disorders identified were anxiety (30\%), followed by depressive and somatoform disorders (17\% each). Agoraphobia was found to be 


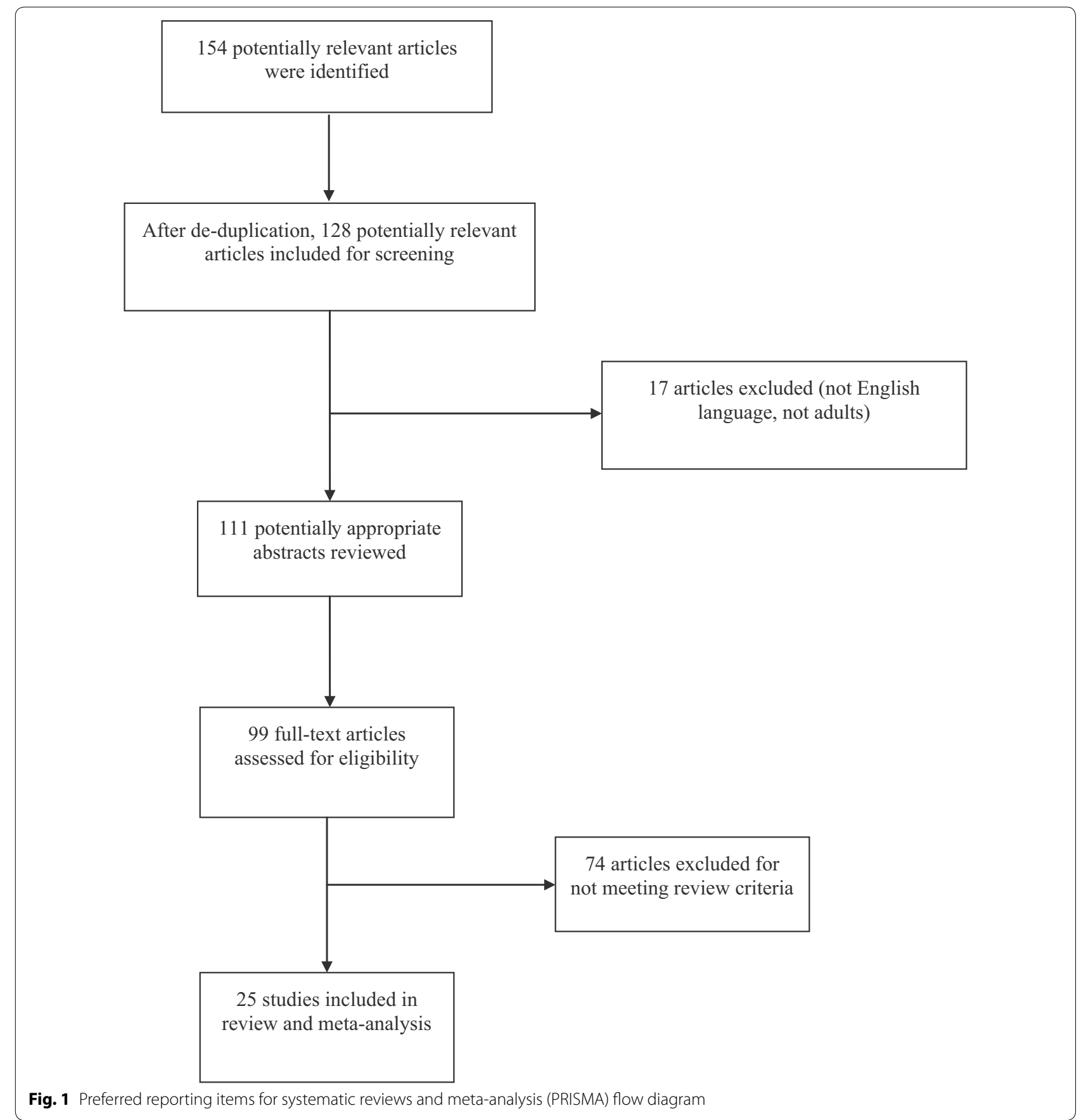

the most frequent anxiety disorder in patients with $\mathrm{CU}$ (15\%).

In 2007, Mehta et al. [23] evaluated the psychiatric illness in psoriasis vulgaris and $\mathrm{CU}$ patients. In $50 \mathrm{CU}$ patients, most common psychiatric co-morbidity was depression (30\%) followed by suicidality (12\%), panic disorder (4\%), obsessive-compulsive disorder (2\%), alcohol abuse and dependence psychotic disorder and mood disorder with psychotic features (2\%). Unfortunately, the total number of patients suffering from any psychiatric disorder was not mentioned.

Similarly, in 2017, Sorour et al. [24] performed an analysis of psychiatric disorders associated with chronic dermatologic diseases among a group of Egyptian patients. This study included 110 patients with CU and anxiety was found in $43.64 \%(n=48)$, depression in $35.45 \%(n=39)$, 
Table 1 Psychiatric comorbidity among chronic urticaria patients: all studies

\begin{tabular}{|c|c|c|c|}
\hline Study & Type of study & Patients & Control group \\
\hline \multicolumn{4}{|c|}{ I. Psychiatric comorbidity in CU patients (studies without a control group) } \\
\hline 1. Juhlin [18] & Cohort study & 330 CU patients & No \\
\hline 2. Pulimood et al. [19] & Cohort study & 20 CU patients & No \\
\hline 3. Picardi et al. [20] & Cohort study & 29 CU patients & No \\
\hline 4. Picardi et al. [21] & Cohort study & 16 CU patients & No \\
\hline 5. Staubach et al. [22] & Cohort study & $100 \mathrm{CU}$ patients & No \\
\hline 6. Mehta et al. [23] & Cohort study & 50 CU patients & No \\
\hline 7. Sorour et al. [24] & Cohort study & 110 CU patients & No \\
\hline \multicolumn{4}{|c|}{ II. Psychiatric comorbidity in CU patients (studies with healthy individuals as a control group) } \\
\hline 8. Uguz et al. [26] & Case-control & 89 CU patients & 60 individuals \\
\hline 9. Atefi et al. [25] & Case-control & 30 CU patients & 30 individuals \\
\hline 10. Chu et al. [17] & Case-control & 177,879 CU patients & 996,356 individuals \\
\hline 11. Staubach et al. [27] & Case-control & 100 CU patients & 96 individuals \\
\hline 12. Ozkan et al. [28] & Case-control & 84 CU patients & 75 individuals \\
\hline 13. Lapi et al. [29] & Case-control & 3489 CU patients & $1,714,746$ individuals \\
\hline \multicolumn{4}{|c|}{ III. Psychiatric comorbidity in CU patients (studies with control subjects other than healthy individuals) } \\
\hline 14. Calıkusu et al. [31] & Case-control & 31 CU patients & $\begin{array}{l}31 \text { patients with psycho- } \\
\text { genic excoriation (PE) }\end{array}$ \\
\hline 15. Yang et al. [30] & Case-control & 75 CU outpatients & 133 patients with tinea pedis \\
\hline \multicolumn{4}{|c|}{ IV. Comorbidity with specific psychiatric disorders in CU patients } \\
\hline 16. Demet et al. [33] & Cohort study & $7 \mathrm{CU}$ & No \\
\hline 17. Sukan et al. [34] & Case-control & 50 CU patients, 50 vitiligo patients & 50 individuals \\
\hline 18. Chung et al. [32] & Case-control & 100 CU patients & 60 patients with allergy \\
\hline 19. Bashir et al. [36] & Cohort study & 3 CU patients & No \\
\hline 20. Tuna et al. [35] & Case-control & 130 CU patients & 100 individuals \\
\hline \multicolumn{4}{|c|}{ V. Case reports-case series_-psychiatric interventions in CU patients } \\
\hline 21. Hashiro [37] & Case report & 1 CU patient with anxiety-depressive disorder & No \\
\hline 22. Gupta et al. [39] & Case series & 5 patients with PTSD diagnosed with CU & No \\
\hline 23. Gupta et al. [38] & Case series & 2 CU patients with panic disorder & No \\
\hline \multicolumn{4}{|l|}{ VI. Other studies } \\
\hline 24. Seyhan et al. [40] & Cohort study & 636 dermatologic patients & No \\
\hline 25. Perugi et al. [41] & Cohort study & 347 patients with bipolar disorder & No \\
\hline
\end{tabular}

Table 2 Psychiatric comorbidity in chronic urticaria patients-studies not including a control group

\begin{tabular}{lcc}
\hline & CU patients & $\begin{array}{c}\text { CU patients with psychiatric } \\
\text { comorbidity }\end{array}$ \\
\hline $\begin{array}{l}\text { Juhlin [18] } \\
\text { Cohort study }\end{array}$ & 330 & 53 \\
Pulimood et al. [19] & 20 & 15 \\
$\quad$ Cohort study & 29 & 10 \\
$\begin{array}{l}\text { Picardi et al. [20] } \\
\text { Cohort study }\end{array}$ & 100 & 48 \\
Staubach et al. [22] \\
$\quad$ Cohort study & 479 & 126 \\
Total & & $26.3 \%$ \\
Pooled prevalence & &
\end{tabular}


Table 3 Psychiatric comorbidity in chronic urticaria (CU) patients-studies including a control group

\begin{tabular}{|c|c|c|c|c|}
\hline & \multicolumn{2}{|c|}{ Chronic urticaria patients } & \multicolumn{2}{|c|}{ Control individuals } \\
\hline & All & $\begin{array}{l}\text { With psychiatric } \\
\text { comorbidity }\end{array}$ & All & $\begin{array}{l}\text { With psychiatric } \\
\text { comorbidity }\end{array}$ \\
\hline \multicolumn{5}{|l|}{ Uguz et al. [26] } \\
\hline Case-control study & 89 & 44 & 60 & 8 \\
\hline \multicolumn{5}{|l|}{ Atefi et al. [25] } \\
\hline Case-control study & 30 & 19 & 30 & 14 \\
\hline \multicolumn{5}{|l|}{ Chu et al. [17] } \\
\hline Case-control study & 177,879 & 56,195 & 996,356 & 45,449 \\
\hline \multirow[t]{2}{*}{ Total } & 177,998 & 56,258 & 996,446 & 45,471 \\
\hline & $119^{\#}$ & $63^{\#}$ & $90^{\#}$ & $22^{\#}$ \\
\hline \multirow[t]{2}{*}{ Pooled prevalence } & & $31.6 \% *$ & & $4.6 \% *$ \\
\hline & & $52.9 \%^{\#, * *}$ & & $24.4 \%^{\#, * *}$ \\
\hline
\end{tabular}

* Pearson's $x^{2}$ : $p$-value $<0.001$

** Pearson's $x^{2}: p$-value $=0.006$

\# After excluding Chu et al. study

suicide ideation in 21 (19.09\%), sleep disorders in 57 (51.82\%), obsessive-compulsive diseases in $3(2.73 \%)$ and finally sexual disorders in 24 of them (21.82\%).

\section{Psychiatric comorbidities in patients with chronic urticaria (studies with healthy individuals as a control group) \\ Description of studies}

Three studies examined the comorbidity of CU with psychiatric clinical entities and included a control group with "healthy" individuals $[17,25,26]$. As "healthy individuals" were defined those who were not suffering from CU. After meta-analyzing these three studies, among 177,998 CU patients, 56,258 had psychiatric comorbidity (31.61\%). Among 996,446 healthy individuals (control group), 45,471 were found to suffer from at least one psychiatric disorder (4.6\%) (Table 3).

There were two studies where the number of healthy individuals with psychiatric comorbidity was not determined [27, 28] and another one that did mention the number of $\mathrm{CU}$ patients or healthy individuals suffering from specific psychiatric diseases, but not the actual number after adjusting for psychiatric comorbidities [29].

\section{Main findings}

The study by Uguz et al. [26] included a total of $89 \mathrm{CU}$ patients and a control group composed of 64 hospital personnel and their relatives. Forty-four (49.4\%) of the patients with $\mathrm{CU}$ met the criteria for at least one psychiatric disorder in contrast to the control group where only eight patients (12.5\%) suffered from at least one psychiatric disorder.
Atefi et al. [25] aimed to compare the psychological scales in patients with $\mathrm{CU}$ with non-dermatological individuals. Thirty patients with $\mathrm{CU}$ participated in this study, and 30 individuals without any skin related disorders were enrolled as controls. Nineteen $(63.3 \%)$ out of the $30 \mathrm{CU}$ patients suffered from psychiatric disorders, whereas this was the case in $14(46.6 \%)$ in the control group.

In 2017 Chu et al. [17] investigated the prevalence, incidence, and comorbidities of $\mathrm{CU}$ in the general population of Taiwan. The observed prevalence of psychiatric disorders among $177,879 \mathrm{CU}$ patients was $8.53 \%(\mathrm{n}=56,195)$. Among 996,356 healthy individuals (control group), 45,449 (4.56\%) were found to suffer from at least one psychiatric disorder.

Staubach et al. [27] in 2006 determined what aspects of life quality are affected and characterize the factors that influence the QoL in CU patients. The study included 100 patients with $\mathrm{CU}$ and 96 healthy subjects. Forty-eight $\mathrm{CU}$ patients were found to suffer from at least one psychiatric disorder. However, the corresponding number of healthy individuals was not mentioned.

Similarly, the study by Ozkan et al. [28] included $84 \mathrm{CU}$ patients and 75 healthy controls. In this study, $60 \%$ of CU patients suffered from at least one psychiatric disorder, but the corresponding number among the healthy control group was not mentioned.

Last but not least, Lapi et al. [29] obtained information on the epidemiology of $\mathrm{CU}$ in Italy. The data source was the Health Longitudinal Patient Database. Among $3489 \mathrm{CU}$ patients and 1,714,746 patients without $\mathrm{CU}$, one was found to suffer from excessive alcohol consumption 
Table 4 Pooled prevalence of psychiatric disorders among chronic urticaria (CU) patients, categorized according to Diagnostic and Statistical Manual of Mental Disorders (DSM-5) (data listed for disorders referred in at least two different published articles)

\begin{tabular}{|c|c|c|c|c|c|}
\hline \multirow[t]{2}{*}{ DSM-5 classification } & \multicolumn{2}{|l|}{$\begin{array}{l}\text { Patients } \\
\text { N/all CU patients }\end{array}$} & \multicolumn{2}{|l|}{$\begin{array}{l}\text { Control }^{\mathrm{a}} \\
\text { N/all healthy controls }\end{array}$} & \multirow[t]{2}{*}{ p-value } \\
\hline & Prevalence per study & Pooled prevalence (\%) & Prevalence per study & Pooled prevalence (\%) & \\
\hline $\begin{array}{l}\text { Sleep-wake disorders (e.g., } \\
\text { insomnia disorder, restless legs } \\
\text { syndrome) }\end{array}$ & $\begin{array}{l}57 / 110[24] \\
31 / 130[35]\end{array}$ & $88 / 240(36.7 \%)$ & $12 / 100[35]$ & (12\%) based only of ref. [35] & $<0.001$ \\
\hline $\begin{array}{l}\text { Anxiety disorders (e.g. generalized } \\
\text { anxiety disorder, phobias) }\end{array}$ & $\begin{array}{l}30 / 100[22] \\
2 / 50[23] \\
48 / 110[24] \\
24 / 30[25] \\
39 / 89[26] \\
25 / 100[27] \\
10 / 84[28] \\
4 / 31[31] \\
15 / 50[34]\end{array}$ & $197 / 644(30.6 \%)$ & $\begin{array}{l}13 / 30[25] \\
7 / 64[26]\end{array}$ & $20 / 94(21.3 \%)$ & $0.16^{\mathrm{b}}$ \\
\hline $\begin{array}{l}\text { Mood disorders (major depressive } \\
\text { disorder, dysthymic disorder) }\end{array}$ & $\begin{array}{l}12 / 20[19] \\
17 / 100[22] \\
16 / 50[23] \\
39 / 110[24] \\
15 / 30[25] \\
18 / 89[26] \\
11 / 100[27] \\
36 / 84[28] \\
6 / 31[31] \\
23 / 50[34] \\
2 / 3[36]\end{array}$ & 195/664 (29.4\%) & $\begin{array}{l}5 / 30[25] \\
3 / 64[26]\end{array}$ & $8 / 94(8.5 \%)$ & 0.001 \\
\hline $\begin{array}{l}\text { Trauma and stressor-related dis- } \\
\text { orders (e.g. posttraumatic stress } \\
\text { disorder, adjustment disorder) }\end{array}$ & $\begin{array}{l}5 / 100[22] \\
1 / 31[31] \\
34 / 100[32]\end{array}$ & $40 / 231(17.3 \%)$ & - & - & - \\
\hline $\begin{array}{l}\text { Somatic symptom and related } \\
\text { disorders (e.g., somatic symptom } \\
\text { disorder, conversion disorder) }\end{array}$ & $\begin{array}{l}17 / 100[22] \\
21 / 30[25] \\
11 / 100[27] \\
5 / 84[28]\end{array}$ & $54 / 314(17.2 \%)$ & $12 / 30[25]$ & (40\%) based only of ref. [25] & $0.020^{c}$ \\
\hline $\begin{array}{l}\text { Obsessive-compulsive and related } \\
\text { disorders }\end{array}$ & $\begin{array}{l}4 / 100[22] \\
1 / 50[23] \\
3 / 110[24] \\
23 / 89[26] \\
3 / 31[31] \\
2 / 7[33]\end{array}$ & $36 / 387(9.3 \%)$ & $1 / 64[26]$ & (1.6\%) based only of ref. [26] & 0.045 \\
\hline $\begin{array}{l}\text { Substance-related and addic- } \\
\text { tive disorders (e.g., alcohol use } \\
\text { disorder) }\end{array}$ & $\begin{array}{l}5 / 100[22] \\
1 / 50[23]\end{array}$ & $6 / 150(4 \%)$ & - & - & - \\
\hline
\end{tabular}

$N$ number of patients with psychiatric disorders

*Comparisons based on Pearson's $x^{2}$

a Only studies comparing the prevalence of psychiatric disorders in CU patients and healthy controls are listed

b Non-statistically significant result attributed to the high prevalence of psychiatric entities among control group in reference 25 (46.6\%)

c Statistically significant result attributed to the high prevalence of psychiatric entities among control group in reference 25 (46.6\%)

(vs. 2307), eight patients reported insomnia (vs. 5076), 74 patients were suffering from anxiety, dissociative and somatoform disorders (vs. 32,064), and four of them had an acute reaction to stress (vs. 1582), respectively. Since more than one of these symptoms or disorders may coexist in the same patient, it is not clear, and it is not mentioned how many patients suffered from at least one psychiatric disorder so that the relative prevalence could be calculated. 
III. Psychiatric comorbidities in patients with chronic urticaria (studies with control subjects other than healthy individuals)

\section{Description of studies}

Two studies reported comorbidity of $\mathrm{CU}$ with at least one psychiatric clinical disorder and utilized a control group with subjects other than healthy individuals [30, 31].

\section{Main findings}

Calikusu et al. [31] compared 31 patients diagnosed with psychogenic excoriation (PE) and 31 patients with $\mathrm{CU}$ (control group) in terms of comorbid psychiatric disorders. There was a statistically significant difference between the two groups in terms of current major depressive disorder (PE group 58.1\%, control group 6.5\%, $\mathrm{p}<0.01$ ). In the PE group, $45.2 \%$ of subjects were diagnosed with obsessive-compulsive disorder (OCD), while the rate of OCD was $3.7 \%$ in the CU patients $(\mathrm{p}<0.01)$.

In 2005 Yang et al. [30] studied a total of 75 consecutive outpatients with CU and 133 consecutive patients with tinea pedis who served as the control group. Compared with controls, cases with $\mathrm{CU}$ experienced significantly more severe somatic and psychosomatic symptoms.

\section{Comorbidities with specific psychiatric disorders in patients with chronic urticaria Description of studies}

Five studies investigated the CU comorbidity with specific psychiatric disorders [32-36].

\section{Main findings}

Demet et al. [33] performed a study to determine the prevalence of obsessive-compulsive disorder (OCD) in patients attending the outpatient department of dermatology. Of 166 patients, 41 (24.7\%) met the DSM criteria for OCD, among whom 2 had CU (4.9\%), while five patients (4\%) had CU in the non-OCD group.

Sukan et al. [34] compared 50 vitiligo patients and 50 $\mathrm{CU}$ patients with 50 healthy controls to assess sexual dysfunctions. The prevalence of sexual dysfunctions in the vitiligo group were $62.5 \%(\mathrm{n}=15)$ in females and $11.5 \%(\mathrm{n}=3)$ in males; in the CU group, it was $70.5 \%$ $(n=24)$ in females and $31.2 \%(n=5)$ in males; the rates in the control group were $16 \%(\mathrm{n}=4)$ in both females and males. The rates of dysthymic disorders and generalized anxiety disorders were higher in the CU group (46\% and $30 \%$, respectively) than the vitiligo group ( $26 \%$ and $6 \%$, respectively).

Chung et al. [32] investigated the relationship between post-traumatic stress disorder (PTSD), stress, psychiatric comorbidity, and personality traits among patients with CU. One hundred patients with CU participated in the study. Sixty patients with allergy (type I hypersensitivity) constituted the control group. Among them, $34 \%$ of the $\mathrm{CU}$ patients and $18 \%$ of the allergic individuals met the diagnostic criteria for PTSD. Patients with CU were 1.89 times more likely to have a current diagnosis of PTSD than allergic individuals.

Bashir et al. [36] aim to determine the frequency of depression among 114 adult individuals that visited dermatology outpatients' clinics. Two out of $3 \mathrm{CU}$ patients were diagnosed with depression (66.6\%).

Finally, in 2016 Tuna et al. [35] included 130 patients with CU and 100 healthy controls in a study that aimed to determine the prevalence and severity of restless leg syndrome (RLS) and to compare the quality of sleep of $\mathrm{CU}$ patients with and without RLS. The prevalence (23.8\%) of RLS in the CU group was significantly higher than the control group (12\%). Similarly, the frequency of poor sleep quality in the CU group was significantly higher than in the control group.

\section{Case reports-case series-psychiatric interventions in patients with CU Description of studies}

Very little is known about the effects various psychiatric interventions may have on urticaria, including both psychopharmacological and psychotherapeutic interventions. One case report [37] and two case series [38, 39] were found to mention that treatment of psychiatric disorders with either anti-depressants or anti-anxiety drugs may result in improvement of urticaria.

\section{Main findings}

In 1995 Hashiro [37] presented a case of a patient with urticaria treated with various combinations of antihistamines (and "antiallergics") with an only slight improvement of her condition. She presented wheals and erythema despite taking these drugs. Three kinds of psychological tests showed that the patient was highly anxious and depressive, so she was additionally treated with psychotropics and psychotherapy. After a month, the symptoms of both urticaria and psychiatric disorder began to disappear.

The same year Gupta et al. [38] presented two patients with a history of severe $\mathrm{CU}$ (corticosteroids were needed regularly to control it) occurring in conjunction with a panic disorder. Both urticaria and panic disorder responded favorably to a course of the selective serotonin reuptake inhibitor antidepressants, fluoxetine and sertraline.

In 2012 Gupta et al. [39] reported five patients with PTSD suffering from $\mathrm{CU}$ with or without angioedema. 
In all patients, CU improved after treatment of the PTSD with trauma-focused psychotherapy intervention.

\section{Other studies \\ Main findings}

Seyhan et al. [40] studied psychiatric morbidity among patients with skin disorders in a dermatology clinic. Of the 636 patients involved in the study, 15.3\% were diagnosed with at least one psychiatric disorder. From those with psychiatric morbidity, 25 had CU (25.8\%).

In 2014 Perugi et al. [41] explored the prevalence and clinical correlates of general medical conditions (GMC) in a large consecutive sample of patients with Bipolar Disorder. The study sample comprised of 347 patients who met DSM-IV-TR criteria for bipolar disorder type I, bipolar disorder type II, or cyclothymic disorder. Among them, 32 patients $(9.2 \%)$ were found to suffer from CU.

\section{Discussion}

This systematic review and meta-analysis has investigated the psychiatric comorbidity among patients with CU. The results suggest clearly that the prevalence of psychiatric disorders in patients with CU is significantly higher than in healthy subjects. The overall prevalence of any psychopathology among $\mathrm{CU}$ patients after pooling all available data from the meta-analyzed studies was estimated to be $31.6 \%$. This suggests that screening for psychological difficulties/mental health problems among CU patients is a necessity. What is not clear, though, is which might be the benefit of a multidisciplinary approach (allergists/ dermatologists and psychiatrists) to control urticaria with or without any additional psychiatric medication or any relevant non-pharmacological psychological or psychiatric intervention. Last but not least, it is not clear if any potential pathophysiological pathways could explain coexistence or sequel from one entity to the other.

\section{Overall completeness and applicability of evidence}

Searching the literature is more than evident that there is limited data about psychiatric comorbidity and factors associated with psychiatric disorders in patients with $\mathrm{CU}$. In 1987 Lyketsos et al. [42] mentioned that patients with CU could develop psychiatric comorbidities. Since then, only a few studies have demonstrated this hypothesis. The development or simply, co-existence of psychopathology and $\mathrm{CU}$ seems to be more complicated since the frequency of the differently classified psychiatric disorders does not follow the global prevalence of these disorders in the general population. According to the World Health Organization [43], the global health estimates support depression as the leading mental disorder followed by anxiety. Similar results have been recently demonstrated by Tat TS [44]. However, in this meta-analysis, the sleep-wake disorders were found to be the most common, followed by anxiety and lastly by mood disorders, including depression (Table 4). There could be psychological reasons (i.e., behavioral/social rather than biological factors) why this makes intuitive sense, for instance, sleep disturbances are expected to be more common than in general population due to itch/discomfort, anxiety due to self-consciousness of appearance or uncertainty of flare-ups. It remains to be further examined whether there is a predisposition of $\mathrm{CU}$ to these psychiatric entities, or if there are particularities among $\mathrm{CU}$ patients that may influence these estimates [CU-specific symptoms and signs, age, ethnicity, gender (most common in females), or if there are just random estimates from the existing studies].

Although psychiatric disorders have been expressed as a potential risk factor of $\mathrm{CU}[3,8,24,45,46]$, the underlying pathomechanisms that may connect these entities have not been clarified yet [11, 45, 47, 48]. Reports suggest that $\mathrm{CU}$ may emerge through interactions between the immune and the central nervous system (CNS) [49]. A 'brain-skin' connection with local neuro-immunoendocrine circuitry may underlie the pathogenesis of several allergic and inflammatory skin diseases triggered or aggravated by stress $[50,51]$. There seems to be a variety of different pathways, not necessarily mutually exclusive, that could explain how inflammatory dysregulation can affect the brain [52-56]. Peripheral inflammation markers affect the brain without passing the blood-CNS barrier. Proinflammatory cytokines activate the tryptophan-kynurenine pathway, regulate serotonin production together with $\mathrm{N}$-methyl-D-aspartate (NMDA) glutamate receptor activity and may also indirectly affect dopamine regulation [57]. The increased inflammation in autoimmune entities may also increase the permeability of the blood-CNS barriers, making the brain vulnerable to cytokines or auto-antibodies. Psychiatric and neurological symptomatology can be induced by brain-reactive antibodies [57-61]. Stress has a vital role in the activation of the immune system and skin by influencing the functions of $\mathrm{T}$ cells [57] and can cause abnormal tension on the autonomic nervous system which in turn affects the levels of histamine in plasma and probably in cells [59]. Stress was found to be associated with the activation of the sympathetic, adrenomedullary system, and the HPA axis [57, 62]. In acute stressful situations, both the adrenocortical and medullary systems are activated, leading to an enhanced release of cortisol and catecholamines. However, chronic stress may induce hyporesponsiveness of the HPA axis, whereby cortisol secretion is attenuated and leads to increased secretion of inflammatory cytokines that are typically counter-regulated by cortisol [62]. Thus, chronic stress can result in some 
inflammatory disorders, such as $\mathrm{CU}$, in which degranulation and mediator release from mast cells and possibly basophils have also been reported [57, 62]. Insomnia itself may further disturb the circadian rhythm of the secretion of cortisol and precipitate the vicious cycle of CU.

A bidirectional relationship between the brain and the immune system also exists [59]. Stress has been shown to suppress or even activate immune system functioning $[59,60]$. Stress also has been associated with worse outcomes in immune-related disorders, including infectious diseases and cancer. This fact is of great clinical importance and underlines the vital effect of stress on the immune system and the clinical expression of the disorders $[59,60]$. Conversely, several lines of evidence suggest that immune system activation is associated with the development of behavioral symptoms similar to those seen in the context of chronic stress [62]. Nowadays, the activation of the immune system is thought to be one of the main pathophysiological causes of mental health disorders [53, 57, 62]. Abnormal functioning of either HPA axis or the inflammatory process disrupts feedback regulation of both immune and neuroendocrine systems contributing to the development of immune-neuro-psychiatric disorders.

One implication from the pathogenesis model mentioned above is that if the level of stress can be controlled, the impact on the whole chain of biological mechanisms can also be reduced. From this point of view, Gupta et al. [38], as well as Hashiro et al. [37], treated their patients with antiallergic, psychotropic drugs (benzodiazepines and antidepressants) and psychotherapy. The urticaria in all three cases improved. It has not been reported that benzodiazepines and antidepressants have direct effects on the suppression of histamine release. Therefore, it is suggested that neuropeptides from nerve endings may also evoke urticaria. What if patients with CU could benefit from psychiatric treatment? It seems that psychotropic therapy may affect $\mathrm{CU}$ control. This evidence needs to be explored.

\section{Strengths and limitations of this review}

The limitations of this work stem primarily from: (i) the lack of intervention studies and randomized control trials; (ii) the heterogeneity of study designs or inappropriate designs (including lack of psychological/ psychopathological epidemiological data in the control group, lack of a control group or even comparisons with non-healthy individuals that were used as a control groups); (iii) the heterogeneity among recruited patients and control groups (including age, gender, demographic characteristics, ethnicity, CU severity); (iv) small study sample sizes; (v) different psychometric tools and diagnostic criteria used and, finally, (vi) the lack of studies in other than English language.

\section{Authors' conclusion}

Current hypotheses, based on the biopsychosocial model, indicate that many organic diseases are multifactorial, contributing to both disease onset and outcome. Whether psychiatric disorders are a potential cause or a consequence of $\mathrm{CU}$ remains to be resolved. Further studies should examine the influence of psychiatric disorders and determine the effects of appropriate psychiatric interventions, including pharmacotherapy or psychotherapy on the course of CU. Till then, our findings call for mental health evaluations of patients with $\mathrm{CU}$ in routine clinical practice. Patients with $\mathrm{CU}$ should be referred, investigated and treated for any mental health disease, a cooperation between allergists/dermatologist and psychiatrists that, teleologically, is expected to improve both health and QoL in these patients.

\section{Abbreviations \\ CU: chronic urticaria or chronic idiopathic urticaria or chronic spontaneous urticaria; DSM: Diagnostic and Statistical Manual; PNS: peripheral nerve sys- tem; ICD: international classification of diseases; OCD: obsessive-compulsive disorder; RLS: restless leg syndrome; PTSD: post-traumatic stress disorder; CNS: central nervous system; HPA axis: hypothalamic-pituitary-adrenocortical axis.}

\section{Acknowledgements}

The authors would like to acknowledge Chioti Afroditi for helping with literature review and article organization.

\section{Authors' contributions}

Both authors performed literature search of electronic databases, screened articles for eligibility based on the inclusion criterion and assessed the risk of bias, reviewed and extracted information from the eligible full-text articles and contributed to the analysis of the results and the writing of the manuscript. Both authors read and approved the final manuscript.

\section{Funding}

This research did not receive any specific grants from funding agencies in the public, commercial, or not-for-profit sectors.

Availability of data and materials

All data generated or analyzed during this study are included in this published article.

\section{Ethics approval and consent to participate}

The protocol of this systemic review has been registered on PROSPERO with registration number CRD42019122811.

\section{Consent for publication}

Not applicable.

\section{Competing interests}

The authors declare that they have no competing interests.

\section{Author details}

${ }^{1}$ Department of Psychiatry, 251 Hellenic Airforce V. A. General Hospital, Athens, Greece. ${ }^{2}$ Department of Allergy and Clinical Immunology, 424 General Military Training Hospital, 11 Eleftheriou Benizelou Street, Kalamaria, 55 133 Thessaloniki, Greece. 
Received: 21 March 2019 Accepted: 29 July 2019

Published online: 23 August 2019

\section{References}

1. Zuberbier T, Aberer W, Asero R, Abdul Latiff AH, Baker D, Ballmer-Weber $B$, et al. The EAACI/GA²LEN/EDF/WAO guideline for the definition, classification, diagnosis and management of urticaria. Allergy. 2018;73(7):1393-414.

2. Altinoz AE, Taskintuna N, Altinoz ST, Ceran S. A cohort study of the relationship between anger and chronic spontaneous urticaria. Adv Ther. 2014;31(9):1000-7.

3. O'Donnell BF, Lawlor F, Simpson J, Morgan M, Greaves MW. The impact of chronic urticaria on the quality of life. Br J Dermatol. 1997:136(2):197-201.

4. Potocka A, Turczyn-Jablonska K, Merecz D. Psychological correlates of quality of life in dermatology patients: the role of mental health and selfacceptance. Acta Dermatovenerol Alp Pannonica Adriat. 2009;18(2):53-8, $60,62$.

5. Bernstein JA, Lang DM, Khan DA, Craig T, Dreyfus D, Hsieh F. The diagnosis and management of acute and chronic urticaria: 2014 update. J Allergy Clin Immunol. 2014;133(5):1270-7.

6. Czubalski K. Mental disorders in patients suffering from allergy. Psychiatr Pol. 1972;6:277-84.

7. Drunkenmolle R. Neuropsychological findings in chronic urticaria. Dtsch Gesundheitsw. 1971;26:398-401.

8. Koblenzer CS. Psychosomatic concepts in dermatology: a dermatologistpsychoanalyst's viewpoint. Arch Dermatol. 1983;119:501-12.

9. Sperber J, Shaw J, Bruce S. Psychological components and the role of adjunct interventions in chronic idiopathic urticaria. Psychother Psychosom. 1989:51:135-41.

10. Clarke TW. The role of allergy in neuropsychiatry. Psychiatr $Q$. 1940;14:800-8.

11. Caccavale S, Bove D, Bove RM, Montagna LA. Skin and brain: itch and psychiatric disorders. G Ital Dermatol Venereol. 2016;151(5):525-9.

12. Hergüner S, Kiliç G, Karakoç S, Tamay Z, Tüzün U, Güler N. Levels of depression, anxiety and behavioral problems and frequency of psychiatric disorders in children with chronic idiopathic urticaria. Br J Dermatol. 2011;164(6):1342-7

13. Kuo $\mathrm{CL}$, Chen $\mathrm{CY}$, Huang HL, Chen $\mathrm{WL}$, Lee $\mathrm{HC}$, Chang $\mathrm{CY}$, et al. Increased risk of major depression subsequent to a first-attack and non-infection caused urticaria in adolescence: a nationwide population-based study. BMC Pediatr. 2014; 14:181

14. Lindsay K, Goulding J, Solomon M, Broom B. Treating chronic spontaneous urticaria using a brief' whole person' treatment approach: a proof-ofconcept study. Clin Transl Allergy. 2015:5:40.

15. Broom BC. A reappraisal of the role of 'mindbody' factors in chronic urticaria. Postgrad Med J. 2010;86:365-70.

16. Liberati A, Altman DG, Tetzlaff J, Mulrow C, Gøtzsche PC, loannidis JP, et al The PRISMA statement for reporting systematic reviews and meta-analyses of studies that evaluate healthcare interventions: explanation and elaboration. BMJ. 2009;339:b2700.

17. Chu CY, Cho YT, Jiang JH, Lin El, Tang CH. Epidemiology and comorbidities of patients with chronic urticaria in Taiwan: a nationwide populationbased study. J Dermatol Sci. 2017:88(2):192-8.

18. Juhlin L. Recurrent urticaria: clinical investigation of 330 patients. Br J Dermatol. 1981;104:369-81.

19. Pulimood S, Rajagopalan B, Rajagopalan M, Jacob M, John JK. Psychiatric morbidity among dermatology inpatients. Natl Med J India. 1996;9:208-10.

20. Picardi A, Abeni D, Melchi CF, Puddu P, Pasquini P. Psychiatric morbidity in dermatological outpatients: an issue to be recognized. Br J Dermatol. 2000;143(5):983-91.

21. Picardi A, Mazzotti E, Pasquini P. Prevalence and correlates of suicidal ideation among patients with skin disease. J Am Acad Dermatol. 2006:54:420-6.

22. Staubach P, Dechene M, Metz M, Magerl M, Siebenhaar F, Weller K, et al. High prevalence of mental disorders and emotional distress in patients with chronic spontaneous urticaria. Acta Derm Venereol. 2011:91(5):557-61.
23. Mehta V, Malhotra SK. Psychiatric evaluation of patients with psoriasis vulgaris and chronic urticaria. German J Psychiatry, 2007;10(4):104-10.

24. Sorour F, Abdelmoaty A, Bahary M, El Birqdar B. Psychiatric disorders associated with some chronic dermatologic diseases among a group of Egyptian dermatology outpatient clinic attendants. J Egypt Women's Dermatol Soc. 2017;14(1):31-6.

25. Atefi N, Rahimi M, Peyghambari S, Ghourchian S. Psychological status in patients with chronic urticaria. Med J Islam Repub Iran. 2011;25:200-4.

26. Uguz F, Engin B, Yilmaz E. Axis I and axis II diagnoses in patients with chronic idiopathic urticaria. J Psychosom Res. 2008;64:225-9.

27. Staubach P, Eckhardt-Henn A, Dechene M, Vonend A, Metz M, Magerl $M$, et al. Quality of life in patients with chronic urticaria is differentially impaired and determined by psychiatric comorbidity. Br J Dermatol. 2006:154:294-8.

28. Ozkan M, Oflaz SB, Kocaman N, Ozseker F, Gelincik A, Büyüköztürk S, et al. Psychiatric morbidity and quality of life in patients with chronic idiopathic urticaria. Ann Allergy Asthma Immunol. 2007;99:29-33.

29. Lapi F, Cassano N, Pegoraro V, Cataldo N, Heiman F, Cricelli I, et al. Epidemiology of chronic spontaneous urticaria: results from a nationwide, population-based study in Italy. Br J Dermatol. 2016:174:996-1004.

30. Yang HY, Sun CC, Wu YC, Wang JD. Stress, insomnia, and chronic idiopathic urticaria-a case-control study. J Formos Med Assoc. 2005;104:254-63.

31. Calikuşu C, Yücel B, Polat A, Baykal C. The relation of psychogenic excoriation with psychiatric disorders: a comparative study. Compr Psychiatry. 2003;44:256-61.

32. Chung MC, Symons C, Gilliam J, Kaminski ER. The relationship between posttraumatic stress disorder, psychiatric comorbidity, and personality traits among patients with chronic idiopathic urticaria. Compr Psychiatry. 2010;51:55-63

33. Demet MM, Deveci A, Taskin EO, Ermertcan AT, Yurtsever F, Deniz F, et al. Obsessive-compulsive disorder in a dermatology outpatient clinic. Gen Hosp Psychiatry. 2005;27:426-30.

34. Sukan M, Maner F. The problems in sexual functions of vitiligo and chronic urticaria patients. J Sex Marital Ther. 2007;33:55-64.

35. Tuna S, Alan S, Samancı N, Karakaş AA. Is there an association between restless legs syndrome and urticaria? J Korean Med Sci. 2016;31:790-4.

36. Bashir K, Dar NR, Rao SU. Depression in adult dermatology outpatients. J Coll Physicians Surg Pak. 2010;20:811-3.

37. Hashiro M. Psychosomatic treatment of a case of chronic urticaria. J Dermatol. 1995;22:686-9.

38. Gupta MA, Gupta AK. Chronic idiopathic urticaria associated with panic disorder: a syndrome responsive to selective serotonin reuptake inhibitor antidepressants? Cutis. 1995;56:53-4.

39. Gupta MA, Gupta AK. Chronic idiopathic urticaria and post-traumatic stress disorder (PTSD): an under-recognized comorbidity. Clin Dermatol. 2012;30:351-4

40. Seyhan M, Aki T, Karincaoglu Y, Ozcan H. Psychiatric morbidity in dermatology patients: frequency and results of consultations. Indian J Dermatol. 2006;51:18-22.

41. Perugi G, Quaranta G, Belletti S, Casalini F, Mosti N, Toni C, et al. General medical conditions in 347 bipolar disorder patients: clinical correlates of metabolic and autoimmune-allergic diseases. J Affect Disord. 2015;170:95-103.

42. Lyketsos CG, Lyketsos GC, Richardson SC, Beis A. Dysthymic states and depressive syndromes in physical conditions of presumably psychogenic origin. Acta Psychiatr Scand. 1987;76:529-34.

43. World Health Organization. Depression and other common mental disorders: global health estimates. Geneva: World Health Organization; 2017. Licence: CC BY-NC-SA 3.0 IGO.

44. Tat TS. Higher levels of depression and anxiety in patients with chronic urticaria. Med Sci Monit. 2019;25:115-20.

45. Hein UR, Henz BM, Haustein UF, Seikowski K, Aberer W, Lautenschlager S, et al. Correlation between chronic urticaria and depression/somatization disorder. Hautarzt. 1996;47:20-3

46. Sheehan-Dare RA, Henderson MJ, Cotterill JA. Anxiety and depression in patients with chronic urticaria and generalized pruritus. Br J Dermatol. 1990;123:769-74

47. Consoli SG. Psychological factors in chronic urticaria. Ann Dermatol Venereol. 2003;130(Spec No 1):1s73-7. 
48. Gregoriou S, Rigopoulos D, Katsambas A, Katsarou A, Papaioannou D, Gkouvi A, et al. Etiologic aspects and prognostic factors of patients with chronic urticaria: nonrandomized, prospective, descriptive study. J Cutan Med Surg. 2009;13:198-203.

49. Ben-Shoshan M, Blinderman I, Raz A. Psychosocial factors and chronic spontaneous urticaria: a systematic review. Allergy. 2013;68:131-41.

50. Paus R, Theoharides TC, Arck PC. Neuroimmunoendocrine circuitry of the 'brain-skin connection'. Trends Immunol. 2006;27:32-9.

51. Segerstrom SC, Miller GE. Psychological stress and the human immune system: a meta-analytic study of 30 years of inquiry. Psychol Bull. 2004;130:601-30.

52. Dalman C, Allebeck P, Gunnell D, Harrison G, Kristensson K, Lewis G, et al Infections in the CNS during childhood and the risk of subsequent psychotic illness: a cohort study of more than one million Swedish subjects. Am J Psychiatry. 2008;165:59-65.

53. Eaton WW, Byrne M, Ewald H, Mors O, Chen CY, Agerbo E, et al. Association of schizophrenia and autoimmune diseases: linkage of Danish national registers. Am J Psychiatry. 2006;163:521-8.

54. Jones AL, Mowry BJ, Pender MP, Greer JM. Immune dysregulation and self-reactivity in schizophrenia: do some cases of schizophrenia have an autoimmune basis? Immunol Cell Biol. 2005;83:9-17.

55. Niebuhr DW, Millikan AM, Cowan DN, Yolken R, Li Y, Weber NS. Selected infectious agents and risk of schizophrenia among US military personnel. Am J Psychiatry. 2008;165:99-106.
56. Yolken RH, Torrey EF. Are some cases of psychosis caused by microbial agents? A review of the evidence. Mol Psychiatry. 2008;13:470-9.

57. Dantzer R, O'Connor JC, Freund GG, Johnson RW, Kelley KW. From inflammation to sickness and depression: when the immune system subjugates the brain. Nat Rev Neurosci. 2008;9:46-56.

58. Diamond B, Huerta PT, Mina-Osorio P, Kowal C, Volpe BT. Losing your nerves? Maybe it's the antibodies. Nat Rev Immunol. 2009;9:449-56.

59. Kayser MS, Kohler CG, Dalmau J. Psychiatric manifestations of paraneoplastic disorders. Am J Psychiatry. 2010;167:1039-50.

60. Lennox BR, Coles AJ, Vincent A. Antibody-mediated encephalitis: a treatable cause of schizophrenia. Br J Psychiatry. 2012;200:92-4.

61. Rose NR. The role of infection in the pathogenesis of autoimmune disease. Semin Immunol. 1998;10:5-13.

62. Tsigos C, Chrousos GP. Hypothalamic-pituitary-adrenal axis, neuroendocrine factors and stress. J Psychosom Res. 2002;53:865-71.

\section{Publisher's Note}

Springer Nature remains neutral with regard to jurisdictional claims in published maps and institutional affiliations.
Ready to submit your research? Choose BMC and benefit from:

- fast, convenient online submission

- thorough peer review by experienced researchers in your field

- rapid publication on acceptance

- support for research data, including large and complex data types

- gold Open Access which fosters wider collaboration and increased citations

- maximum visibility for your research: over $100 \mathrm{M}$ website views per year

At BMC, research is always in progress.

Learn more biomedcentral.com/submissions 